\title{
Amazing grace: How sweet the sound of synthesised bagpipes
}

Hannah Pfeil, Abram Hindle, Hazel Campbell

${ }^{1}$ Department of Computer Science, University of Alberta

\section{Abstract}

A bagpipe is a type of wind instrument that contains a melody pipe, which has an enclosed reed called the chanter and other drone pipes. The chanter is the part of the bagpipe that supplies the note, and the air that the pipes are fed is provided by the bag, which is inflated by a blowpipe and driven by the player's arm. The goal of this project was to create a bagpipe using a program called Supercollider. Supercollider is used for audio synthesis. While creating this artificial bagpipe (here on referred to as a 'synth'), it was broken down into four components: the chanter, the base drone, the first tenor drone and the second tenor drone. The chanter has the frequency of the note, the base drone's frequency will be half that of the chanter and the frequency of the tenor drone will be half that of the base drone. This is because of the length of the pipes in relation to each other. In order to create the synth, a sine oscillator was used, and then put through a resonance filter, and then a reverb filter. This was done in order to mimic the echo that sound has when it is forced through a tube, or enclosed space. All four pipes were added together to create the synth. In order to play a song, the synth was put into a pattern so Supercollider could receive an array of notes, which serve as the frequency of the chanter, and then play the song automatically. The notes for Amazing Grace were transcribed into midi-notes and beat durations and these arrays were fed into the pattern to create the song. The synthetic version of Amazing Grace, in terms of frequency and loudness, was then graphed and compared to the graph of a recording of Amazing Grace played on a real bagpipe. There are differences between the two sound files, the most significant being that the real bagpipe has much more variation in terms of loudness. The synthesized bagpipe had a more gradual and subdued noise level, where the natural bagpipe was much more randomized. Taking the comparisons into consideration, Supercollider can be used to create an approximation of a bagpipe, but under scrutiny, the artificial version currently falls short.

Key words:

bagpipes, sound, synthesized, synthesised, supercollider, composed, synthesised bagpipes

Cite as: Hannah Pfeil, Abram Hindle, Hazel Campbell. 2019. Amazing Grace: How Sweet the Sound of Synthesised Bagpipes. Alberta Academic Review, Vol 2 (2) 59-60, WISEST Special Issue (non peer-reviewed), DOI 10.29173/aar65. 
Pfeil et al., 2019
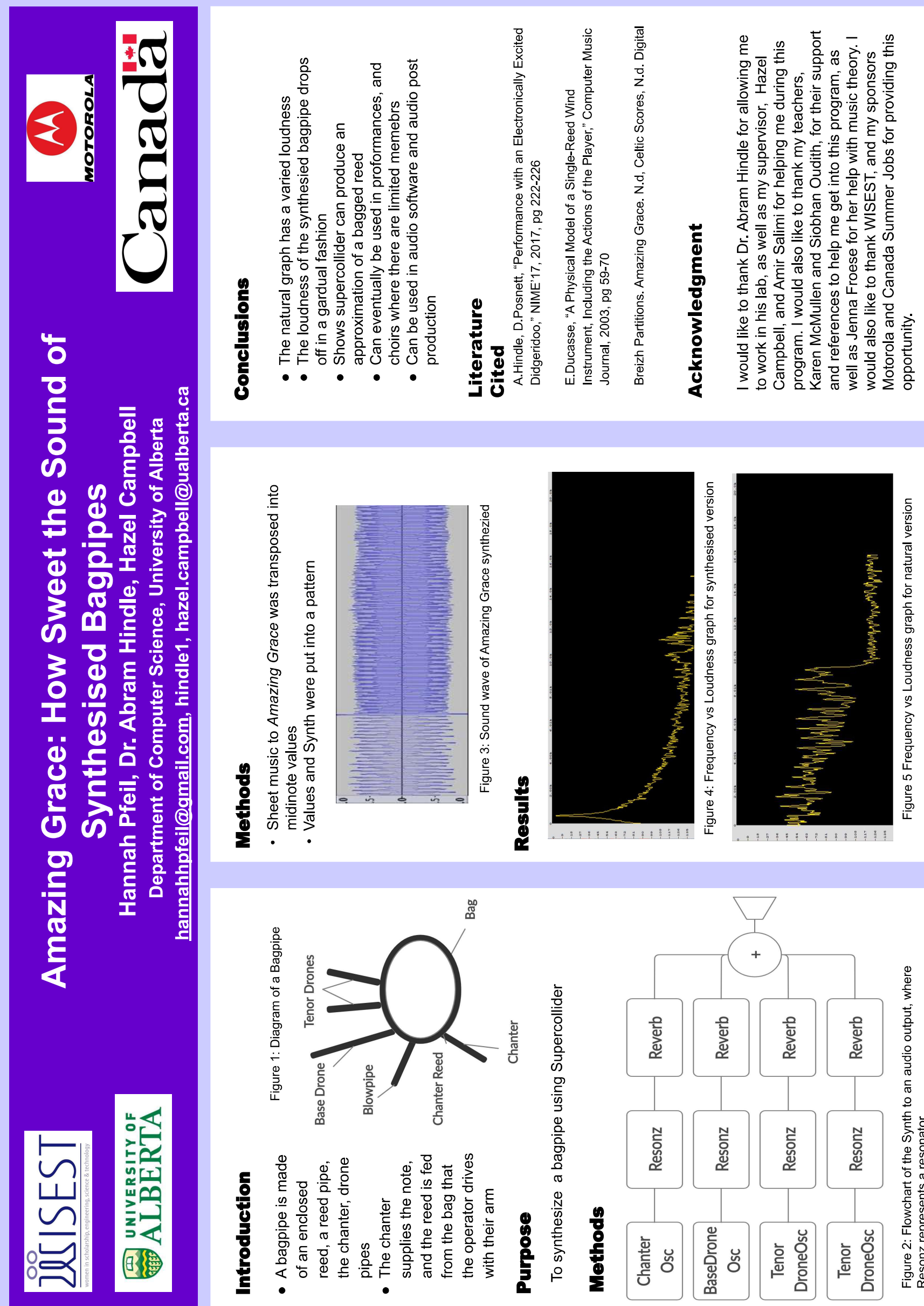

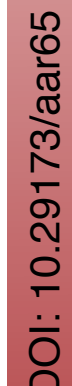
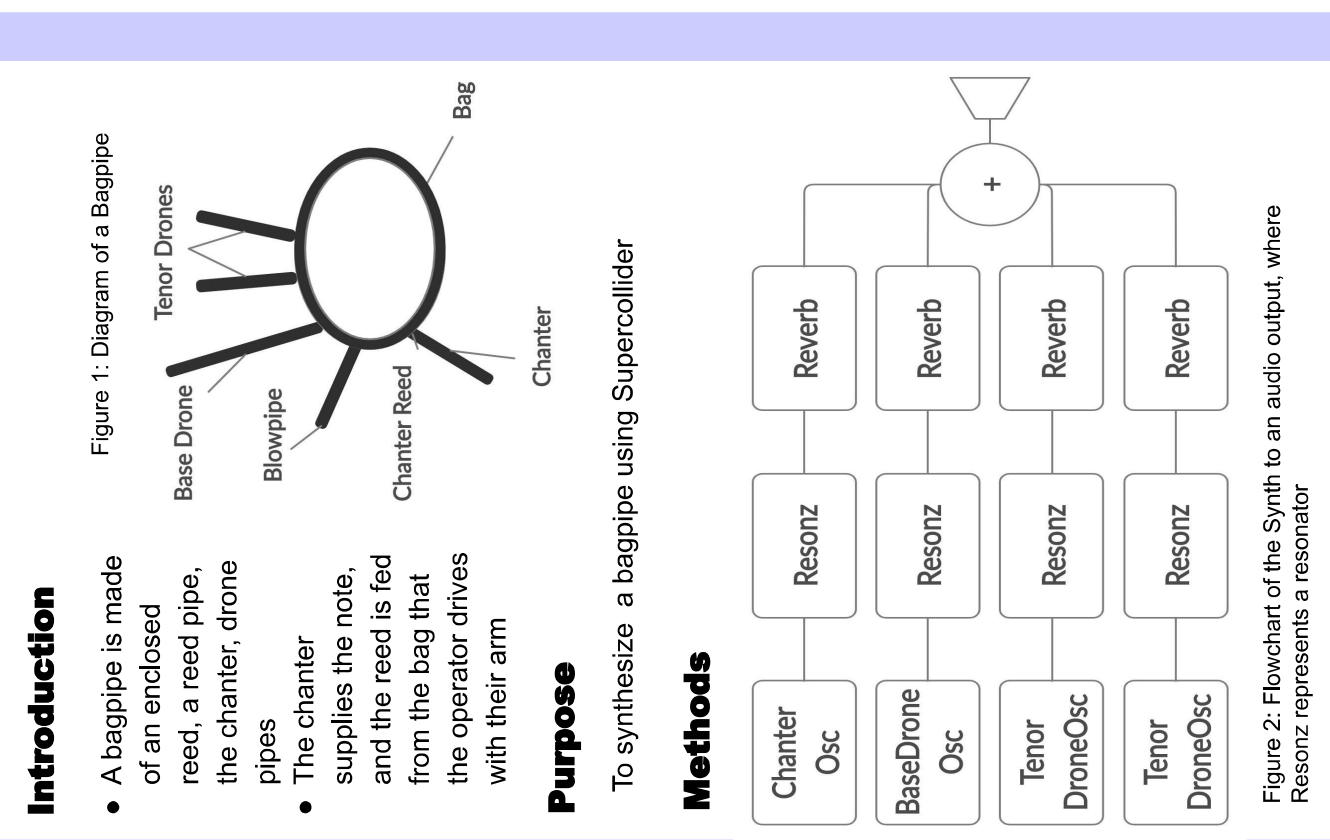